
\title{
25 Research Soure \\ Identification of peach growth regulating-factors and their expression under UVB
}

\section{MingYue Sun}

Shandong Agricultural University https://orcid.org/0000-0002-6227-3106

Shaoxuan Li

qingdao academy of agricultural sc

Xiangguang Meng

Shandong Agricultural University

Li Liu

Shandong Agricultural University

Ning Wang

Shandong Agricultural University

Huajie He

Shandong Agricultural University

Dongmei Li

Shandong Agricultural University

Xiude Chen ( $\nabla$ chenxiude@163.com )

Shandong Agricultural University https://orcid.org/0000-0001-9215-9489

Ling Li

Shandong Agricultural University

\section{Research article}

Keywords: Peach, Growth-regulating factor, Gene family, UVB, GA3, new shoot

Posted Date: August 12th, 2020

DOI: https://doi.org/10.21203/rs.3.rs-32624/v1

License: (c) (i) This work is licensed under a Creative Commons Attribution 4.0 International License.

Read Full License 


\section{Abstract}

Background: Growth-regulating factors (GRFs) are one of the most important plant-specific transcription factors with vital roles in multiple biological processes. GRFs have been identified in a variety of plant species, but a handful of research has addressed GRF genes in peach (Prunus persica).

Results: Here, we report 46 members of the GRF family in four Rosaceae, divided into six subfamilies according to phylogeny, gene structure, and motif composition. We detected three collinear gene pairs generated from peach by whole-genome duplication or segmental duplication, but no tandem repeats were detected. Expression pattern analysis found that most PpGRFs were preferentially expressed in young tissues, At the sametime, multiple types cis-elements were observed in PpGRF promoters, and PpGRFs could positively respond to ultraviolet B-rays (UVB) and gibberellin (GA)treatments at the transcriptional level. Also, the content of $\mathrm{GA}_{3}$ and indole-3-acetic acid (IAA) changed significantly after UVB irradiation, indicating that GRFs might be involved in new shoot development in peach.

Conclusions: This study identified $10 \mathrm{GRF}$ genes in the peach genome and systematically analyzed their properties, thereby providing a foundation for researchers to have a better understanding of this gene family in peach. PpGRF 3, 4, 5, 6, 7, 9, and 10 positive responses to UVB and $\mathrm{GA}_{3}$ signals indicate that they can serve as candidate functional genes to further study how tree potential is regulated in peach.

\section{Background}

Many transcription factors (TFs) have been identified through evolutionary analyses in peach, including AP2,WRKY, MYB, TCP, NAC, NF-Y, and SPL TFs [1-6]. However, the roles of GRFs remain unknown in peach. GRFs are highly conserved in plants, which belongs to a small family of transcription factors that. The first identified GRF gene was described in rice, and was shown to mediate gibberellin-induced stem growth regulation in deepwater rice [7]. evolutionally conserved QLQ and WRC domains are contained in the GRF proteins' N-terminal region [7-9]. The amino acid sequence of the QLQ (GIn, Leu, GIn) domain is somewhat similar to the protein-protein interaction domain of SWI2/SNF2 [10]. The QLQ domain is thought to help GRF proteins to interact with growth regulators (GIFs) to regulate plant growth and development $[8,11]$. The WRC (Trp, Arg, Cys) domain is a plant-specific domain capable of binding to DNA and has a nuclear localization signal and a zinc finger motif $[7,8]$. Also, most GRF proteins have transactivation activity in the $\mathrm{C}$-terminal region and share some less conserved motifs, such as the GGPL (Gly, Gly, Pro, Leu), TQL (Thr, Gln, Leu), and FFD structure domains [11, 12].

As development-related transcription factors, GRFs are highly expressed in tissues that are actively growing and developing, including root and leaf development, stem elongation, meristem maintenance, cell expansion, floral organogenesis, and seed formation [8, 13-15]. Transcription of the GRF gene is regulated by $\mathrm{GA}$. After $\mathrm{GA}_{3}$ treatment of celery cabbage leaves, the transcript levels of several Chinese cabbage GRFs (BrGRF2, 4, 5, 7, 8, 9, 11, 12, 15, and 16) were enhanced [16]. Similarly, $\mathrm{GA}_{3}$ treatment 
enhanced the transcript levels of several rice GRFs (OsGRF1, 2, 3, 7, 8, 10, and 12), while the transcript levels of OsGRF9 decreased [9].

Leaf and cotyledon sizes are increased in Arabidopsis lines overexpressing AtGRF1-3. This finding suggests that the AtGRF1-3 genes have roles in leaf and cotyledon development [8]. In the initial study, only the AtGRF1/2/3 triple mutant had smaller, narrower leaves, and shorter petioles compared to the wild type; however, later reports also demonstrated the first pair of AtGRF3 single-gene mutants. The mean size of leaves was also reduced by $15 \%$, and these changes in organ size resulting from increased GRF expression are due to increased cell size rather than increased cell numbers $[8,17]$.

Interestingly, in contrast to AtGRF1 and AtGRF2, the overexpression of AtGRF5 showed a larger leaf area due to proliferation of cells. Also, the biochemical analysis showed that GRF and GIF combine to form a transcription complex in vivo. By means of a positive feedback regulatory loop, it might be a effective way for the GRF-GIF complex to activate its own transcription, thereby regulating the increase in the number of cells and ultimately controlling leaf size $[14,15,17-19]$. Overexpression of TCPs (TCP4) resulted in reduced transcription levels of GIF, suggesting that they play an intermediary role in the GIFGRF-miR396 regulatory network $[20,21]$.

It has been shown that miRNA396 directly inhibits the expression of GRFs in various plant species through post-transcriptional regulation [11, 23]. The heterologous expression of Ath-miR396a (from Arabidopsis thaliana) in tobacco causes a reduction in the transcription level of the GRF gene in tobacco, accompanied by similar narrow-leaf phenotypes, such as reduced leaf size. Also, Ath miR396a overexpression affects flower development relative to wild type, for inatance, increasing the number of petals, anthers, and carpels, and producing shortened (curved) stamens [24]. Similarly, ectopic expression of Ptc-miR396c (from Populus trichocarpa) resulted in changes in tobacco growth and flower development, accompanied by a decrease in the abundance of NtGRF transcripts [24]. Interestingly, not all GRFs are miR396 target genes. AtGRF1-4 and AtGRF7-9 are miR396 target genes in Arabidopsis, but overexpression of miR396 also inhibits AtGRF6 expression [25, 26]. MiR396 expression is caused by various abiotic stresses, such as high salinity, low temperature, drought stress, and UVB. Several reports indicate that UVB leaf growth inhibition requires GRF1 activity, while miR396/GRF exerts it has an influence on organ growth by regulating (at least partially) the expression of GA metabolic genes [25, 2729].

UVB radiation treatment of Arabidopsis causes the accumulation of DNA damage and limits leaf expansion by inhibiting cell division in proliferating tissues [30]. Reduced maize leaf growth under UVB irradiation is the result of reduced cell production and a shortened growth zone [29]. This inhibition is regulated, at least in part, by transcription factors of the GRF family, some of which are probably regulated by miR396, which modifies GA levels [29]. Soybean plant morphology is substantially altered in response to shade stress. At the same time, under shade stress conditions, the GmGRF9 and GmGRF17 expression levels were strongly suppressed, and GmGRF5 was up-regulated [31]. 
Peach trees are native to China, where they have a cultivation history of about 3000 years [32]. Because peach has a relatively small genome and high economic and nutritional value, it has become the focus of research [33]. The protected cultivation of peach trees is more economical than traditional cultivation, but it limits the growth of fruit trees, yield, and fruit flavor. Among various environmental factors, UVB radiation is an important factor, causing abiotic stress that potentially influences the biological processes of plants [33-35]. Considering greenhouse light conditions, more in-deep researches which are connected with the molecular and metabolic mechanisms under UVB irradiance are now needed.

In this study, a total of $46 \mathrm{GRFs}$ were characterized. We analyzed the phylogenetic relationships among GRF genes, their protein motifs, and gene structure across multiple species, including Arabidopsis thaliana, Maize (Zea mays L.), soybean (Glycine max), rice (Oryza sativa), poplar (Populus trichocarpa), and four Rosaceae fruit species. Moreover, we validated gene expression patterns by transcriptome analysis and quantitative real-time PCR (qRT-PCR) in peach, and identified several candidate GRF genes closely associated with peach. These results will contribute to the further researches of their molecular functions.

\section{Results}

\section{Identification of GRFs in Rosaceae}

The GRF Hidden Markov Model (HMM) configuration file (PF08879 and PF08880) was used to identify GRF members. Furthermore, the SMART tool was used to verify the existence of two characteristic conserved domains (QLQ and WRC) in the candidate genes. After manually removing the incomplete sequences from peach (Prunus persica), European pear (Pyrus communis), strawberry (Fragaria vesca), and apple (Malus domestica), respectively, $46 \mathrm{GRF}$ genes were identified and named according to their position on the chromosome. The GRFs were unevenly distributed across the chromosomes of the four species. The 46 predicted GRF proteins ranged from 187 to 843 amino acid residues in length, and their relative molecular mass varied from 20.70 to $94.27 \mathrm{kDa}$ (Table 1).

\section{Phylogenetic and structural analyses of GRF genes}

To get a better understanding of evolutionary relationships, a neighbor-joining phylogenetic tree was constructed among GRFs from Arabidopsis (9), soybean (22), rice (12) poplar (19), and four Rosaceae species (46). As shown in Fig. 1, $108 \mathrm{GRFs}$ from the different species were divided into six subgroups ( I$\mathrm{VI})$ [31]. The majority [ 23\% (25/108)] of GRF proteins belonged to clade I, 21 to clade II (without AtGRF), 24 to clade III, 15 to clade IV (without OsGRF), 13 to Clade V (without OsGRF), and 10 to clade VI (without GmGRF) (Fig. 1). No species-specific clades were discovered, and every subgroup contained Rosaceae GRFs, which shows that GRF proteins are evolutionarily conserved .

To better explore the evolutionary relationships and predict the function of GRF proteins, we investigated the exon-intron patterns and motif characteristics of GRFs. We found some structural features among 
clades or subclades according to the alignment and motif results. As shown in Fig. 2, a total of 10 conserved motifs of Rosaceae GRFs were found using the MEME online software. Each subgroup has three to nine conserved motifs, and the motif composition is similar within subgroups [36-40]. All of the GRF family members contained motif 1 or motif 2 . Based on such a gene structural analysis, we determined that motif 1 and motif 2 correspond to the WRC and QLQ domains, respectively. Some motifs occur only in certain specific subgroups, which possibly contribute to functional diversity. For instance, motif 6 is unique to subgroup I, and motif 5 is unique to subgroup II, while motifs $3,4,7,9$, and 10 are concentrated in group III. Motifs 3, 4, and 9 are specific to group IV. Motifs 3 and 4 are specific to group VI. We also investigated the structure of GRF gene to further describe their evolutionary trajectory. All GRFs contained conserved QLQ and WRC domains in their N-terminal regions. However, group V has two WRC domains. These results indicate that they contain different exons numbers, varying from 3 to 12 . The gene structures were similar or the same in each subgroup. Overall, phylogenetic relationships are strongly supported by gene structure and motif characteristics.

\section{Chromosomal localization and collinearity analyses of GRF genes}

It's random for the distribution of the GRF genes in the four Rosaceae genomes. Unlike in previous studies, in the four Rosaceae, some chromosomes do not have a GRF gene. In $M$. domestica and $P$. communis, the GRF genes are mainly found on chromosomes 2 and 15 . For $P$. persica and $F$. vesca, the GRF genes were principally distributed on chromosomes 2 and 7, respectively. Additionally, two GRF genes (MdGRF15 and MdGRF16) could not be mapped to any chromosome in the M. domestica genomes (Fig. 3).

Subsequently, in order to further and deeper infer the phylogenetic relationship between peach and other Rosaceae plants, the collinear relationships between three Rosaceae plants and peach (Fig. 3) was analyzed. We found that nine FvGRF genes were collinear with PpGRF genes, followed by MdGRFs (14) and PcGRFs (10). Moreover, the collinear relationship in the peach genome was also examined to elucidate the evolution and origin dynamics of PpGRF genes. Three pairs of PpGRF genes (PpGRF01/08, PpGRF03/07, PpbZIP05/10) have a collinear relationship and were generated by whole-genome duplication (WGD) or segmental duplication (Fig. 3 and Additional File 1: Supplementary Table S1). Furthermore, to determine the selection constraints on the duplicated PpGRF genes, we calculated the non-synonymous/synonymous substitution ratio $(\mathrm{Ka} / \mathrm{Ks})$ for each pair of duplicated genes. The $\mathrm{Ka} / \mathrm{Ks}$ ratios of most PpGRF pairs were less than 1, the evidence shows that these PpGRFs had undergone purifying selection processes (Additional File 1: Supplementary Table S1).

\section{UVB effect on peach leaf growth}


During the whole groeing period, the peach trees were treated with $1.44 \mathrm{Kj} \mathrm{m}^{-2} \mathrm{~d}^{-1} \mathrm{UVB}$ radiation [34]. It can be seen from Fig. 4 that, during the growth period of the new shoot, UVB supplementary light treatment can inhibit the elongation of the new shoot. After harvesting for 120 days (September 7), the new shoot of the UVB group stopped lengthening, while the CK group continued to increase slowly. The average new shoot length $(114.08 \mathrm{~cm})$ was about 1.5 times that of the UVB group $(69.45 \mathrm{~cm})$. The thickness of the new shoot in the CK group increased by $0.3 \mathrm{~cm}$, while the thickness of the new shoot in the treatment group increased by $0.29 \mathrm{~cm}$; the difference was not statistically significant. Based on this finding, this dose of UVB treatment had little effect on the thickness of the new shoot.

\section{Hormone profiles after UVB exposure in peach}

Based on the Previous studies, there have various statistics demonstrated that GRFs play important roles in regulating leaf growth $[8,13-15,41]$. The shoot tip is the growth point of the new shoot, and its hormone content determines the growth of the new shoot. At this facility, the new shoots of peach grow vigorously from June to September, so in this experiment, the shoot tip tissue was taken on August 7 and frozen for storage for hormone content analyses. The results of the hormone content analyses are shown in Fig. 5: Under UVB treatment, the content of IAA varies greatly, and the IAA content of the shoot tip tissue of CK group $(1.978917 \mathrm{pmol} / \mathrm{g})$ is 4.9 times that of UVB treatment group $(0.40339 \mathrm{pmol} / \mathrm{g})$. At the same time, the $\mathrm{GA}_{3}$ content was increased in the UVB treatment group $(0.59663 \mathrm{pmol} / \mathrm{g})$ relative to the CK group $(0.22767 \mathrm{pmol} / \mathrm{g})$. In our hormone levels analysis, IAA content showed a significant difference $(P<0.01)$. The $G_{3}$ content also showed a significant difference $(P<0.05)$. The content of abscisic acid (ABA), jasmonic acid (JA) and trans-Zeatin-riboside (TZR) were not significantly different when comparing CK group. Therefore, we speculate that the UVB-mediated growth inhibition of new shoot is closely related to the IAA and $\mathrm{GA}_{3}$ contents in the shoot tip.

\section{Prediction of the miR396 target site and how its expression changes following UVB exposure}

Differently to Arabidopsis, all GRFs in peach have a sequence that is partially complementary to miR396, which is located at the WRC domain. To delve deeper into the evolutionary relationship among GRFs, we analyzed the WRC structures of all GRFs in the four Rosaceae. According to Fig. 6 , we found that all GRFs of the four Rosaceae contain this part, which is complementary to the miR396 share a bulge at position 7 (counting from the 5 end of the miRNA). From this point of view, the evolution of GRFs in Rosaceae is highly conservative. We have identified a series of differentially expressed miRNAs that have been predicted to be responsive to low-dose UVB. To identify the expression profiles of miRNA396, we utilized transcriptome data of Illumina RNA-Seq reads that were generated and analyzed by Li et al [34]. We found that, in peach, miR396 was down-regulated after UVB irradiation, but this did not reach statistical significance. Also, we isolated another mirR319, which regulates TCP4, induces miR396, and represses 


\section{Analysis of cis-elements in the promoter sequences of PpGRF genes}

To further explore the involvement of the PpGRF genes in light responsiveness, hormone signaling pathways, plant growth and development, their promoter sequences were analysed using PlantCARE software [42]. All cis-elements in the promoter regions of the PpGRF gene family members are shown in Fig. 7. There are at least six commonly occurring light-responsive elements (LREs): AE-box, ATCT-motif, Gbox, GT1-motif, GATA-motif and I-box, which have been demonstrated to be essential for the regulation of light mediated transcriptional activity [43-45]. The results indicated that all 10 PpGRF promoter regions contained two or more LREs among which G-box is the most abundant element (Additional File 3: Supplementary Table S3). In this area we qualificated many other important potential cis-elements, such as GARE motif and P-box (GA responsive element), a CAT-box (cis-acting regulatory element related to meristem expression), AuxRR-core and TGA-element (auxin-responsive element), an RY element (seedspecific regulation) and a TC-rich repeats (defense and stress responsiveness) [46-51]. GARE, P-box, CATbox, AuxRR-core, TGA-element, RY element and TC-rich repeats were distributed within 3, 3, 7, 2, 3, 1 and 5 PpGRF promoter regions, respectively (Additional File 4: Supplementary Fig S1).

\section{Expression pattern of GRF genes in peach}

Although we identified GRF genes in the peach genome, the functions of these genes remain largely unknown. To sum up, GRFs were highly expressed in growing tissues [8]. Next, we studied the patterns of expression about the PpGRFs in root, stem, leaves, the shoot tip and hypocotyl. Except for PpGRF8, these genes were differentially expressed in different tissues (data not shown). Most of these were upregulated, especially in the shoot tip and root (Fig. 8). For example, the expression levels of PpGRF2, 3, 4, $5,6,7,9$, and 10 were the highest in the shoot tip. However, PpGRF1 showed relatively strong expression in the root. Of the analyzed genes, PpGRF3, PpGRF9, and PpGRF10 were more preferentially expressed in the shoot tip; more than 20 times greater than that in other tissues. To further investigate the potential functions of PpGRFs, we also evaluated the effects of $\mathrm{GA}_{3}$ and UVB on the expression of the PpGRF gene (Fig. 9). In abiotic stresses, over half of the total genes were up-regulated, with PpGRF1, 4, 5, 6, 7, and 10 being up-regulated after $\mathrm{GA}_{3}$ treatment; PpGRF2, 3, 4, 5, 6, and 7 were up-regulated after UVB treatment. On the other hand, several PpGRF genes (including PpGRF2, PpGRF6, and PpGRF7) were suddenly downregulated at $9 \mathrm{~h}$ after $\mathrm{GA}_{3}$ treatment (Fig. 9). Also, PpGRF5, PpGRF9, and PpGRF10 were suddenly downregulated at $6 \mathrm{~h}$ after UVB treatment. Based on the expression patterns of PpGRFs, we propose that they likely have multiple functions in regulating growth and responsiveness to abiotic stresses.

\section{Discussion}


Growth regulators are one of the most important plant-specific transcription factors, they play a vital role in plant growth and development, especially in regulating organ size $[14,18,52]$. The GRF gene families in model plants (Arabidopsis, rice, soybean, and poplar) have been identified and described in detail, but little is known about the GRFs in Rosaceae plants $[8,9,11,39]$. In order to have a better understanding of the characteristics and functions of PpGRFs, we studied PpGRF family members by bioinformatics assay with other three Rosaceae plants. Meanwhile, by performing qRT-PCR analysis, plant morphology analysis and hormone content determination to analyze the function of PpGRF. In the current study, we identified 46 GRF members in four representative Rosaceae plants, and found that the number of GRFs in apples is relatively large, perhaps due to a genome-wide replication event in the apple genome [53, 54]. Phylogenetic analysis showed that the four species of Rosaceae GRF could be divided into six groups (IVI) (Fig. 1), which is consistent with the classification of Arabidopsis, soybean, and rice GRFs [8, 9, 11]. The homologous pairs of GRF proteins in the four Rosaceae plants are more common. According to the topology of the phylogenetic tree, we found that some ancestral GRFs existed before the evolutionary divergence of the Rosaceae. By analyzing the GRF domains, we found that all of the speculated GRFs contain QLQ and WRC domains, and the VI subfamily contains two WRC domains. Similar results also exist in the Arabidopsis genome but not in the rice genome, suggesting that a major expansion occurred after the eudicots diverged from the monocots and before the separation of Rosaceae (Additional File 5, Supplementary Table S4). The structures of GRFs belonging to different subgroups in intron and exon structures showed low homology. GRFs of the same subfamily have the same or similar genetic structure, especially the number and length of exons. Also, based on our MEME analysis, each subfamily contains distinct motif arrangements. The differences in these characteristics of subfamilies suggest that GRF members are functionally diverse, which also supports the classifications used in this study $[8,9,11,39]$. The expansion of the GRF family has been achieved mainly through gene duplication, especially largescale duplication (WGD or segment duplication) [11,39]. In this study, three pairs of PpGRF genes were distributed in duplication blocks, which means large-scale, repeats that promote the amplification of the GRF gene family in peach. We also found that the Ka/Ks ratios of all the PpGRFs gene pairs were less than 1 , indicating that they have experienced strong purifying selection.

The excessive vigor of fruit trees will lead to the growth of new shoot, which is not conducive to the storage of assimilates, flower bud differentiation, or the balance between vegetative and reproductive growth [55]. This is one of the critical prevention and control goals in fruit tree cultivation management technology.

Here we found that during the growth period of new shoot, UVB supplementary light treatment can significantly inhibit the growth of new shoot, but has little effect on the changes in the thickness of new shoot. Perhaps UVB inhibits the proliferation of cells in developing leaves. The shoot tip is the growth point of the extension of the new shoot, and its hormone content determines the growth of the new shoot. UVB treatment can significantly reduce the content of IAA and increase the content of $\mathrm{GA}_{3}$ in the shoot tips. Therefore, we infer that the levels of $\mathrm{GA}_{3}$ and IAA in the shoot tips inhibit the growth of the new shoot to a certain extent, and thus regulate tree potential. Studies in maize indicate that the inhibitory 
effect of UVBs on growth is mediated by GRF, some of which might be regulated by miR396, thereby modifying GA levels in the leaf growth zone $[29,30,56]$. To further verify the above hypothesis, we tested the expression of miR396. Under UVB treatment, miR396 maintained a low level of expression in the function leaves and was slightly down-regulated. Unlike in Arabidopsis, miR396 was not highly expressed in the leaf primordia, and then it accumulates gradually with the development of Arabidopsis leaves [26]. At the same time, mirR319 expression was also down-regulated (although not reaching statistical significance). Probably we test the miRNA in the mature leaves, Most of the GRF genes are strongly expressed in the active growth and development tissues, such as shoot tip, root and flower bud, but the mature stem and leaf tissues are weakly erpressed $[8,18]$.

We further explored the expression patterns of GRFs. First, qRT-PCR was used to detect the expression of PpGRFs in various tissues. The spatial expression pattern of PpGRF gene has some important similarities with other plants. Several studies have reported that GRFs transcription levels decrease as organs age. The expression levels of PpGRF2, 3, 4, 5, 6, 7, 9, and 10 in the shoot tip meristem was higher than that in mature tissues, suggesting that these genes play a crucial part in the elongation of the new shoot [57]. PpGRF1 was more highly-expressed in root than in the other tissues, indicating that PpGRFs is also important in root growth [58-60]. A well-documented effect of UVB exposure on plants is the reduction of biomass, and miR396/GRFs exerts its effect on organ growth at least in part by regulating the expression of genes related to GA metabolism $[25,27,28]$. Our study demonstrates that PpGRFs can widely respond to UVB and $\mathrm{GA}_{3}$ treatments at transcriptional levels, with almost half of the members showing induction and a few showing repression.

Notably, most PpGRFs showed a response peak after $9 \mathrm{~h}$ of $\mathrm{GA}_{3}$ treatment, but the response peak after UVB treatment appeared after $6 \mathrm{~h}$. The analysis of cis-elements in the PpGRF promoter sequences found that some members contained multiple types of light responsive elements and GA responsive element, implying the involvement of PpGRFs in different light response and hormone signaling pathways. This shows that there are differences in how GRFs participate in these two signaling pathways. There is much evidence indicating the role of GRFs in various biological processes through interacting with different clients, such as hormone pathways, stomatal behavior, reactive oxygen species balance, and ion transport [61-65]. In Arabidopsis and maize, UVB radiation inhibits leaf growth by decreasing the expression of GRFs $[29,30,56]$. This ultimately leads to a reduced expression of GA biosynthetic genes and increased levels of catabolic transcripts [26, 29, 30, 56]. In addition, the PpGRF promoter regions contain various types of cis-elements, which is helpful to explore the upstream genes of the PpGRFs involved in regulating growth and responsiveness to abiotic stresses. Overall, further study of the function of PpGRFs can reveal the behaviours of PpGRFs during regulate tree growth and development, providing real and reliable information for further studying of the biological functions of PpGRFs.

\section{Conclusions}

In this study, we identified 46 GRFs from four Rosaceae and investigated their phylogenetic classification, protein motifs, and gene structures. By further analysis, GRFs belonging to the same subgroup had 
similar gene structures and motif compositions. Chromosomal localization and collinearity analyses suggested that large-scale duplication contributed to the expansion of the PpGRF family. Physiological experiments and expression analyses have revealed the involvement of PpGRFs under UVB and $\mathrm{GA}_{3}$ signaling and identified some important candidates for regulating the elongation of the new shoot, as well as in abiotic stress response. More research is needed to explore this hypothesis in more detail.This systematic study will advance the understanding of GRF-mediated signal cascades in regulating peach tree development and abiotic stress response. These results will provide a solid and reliable basis for further research on the GRFs in peach.

\section{Methods}

\section{Plant material and treatments}

The peach cultivars (Prunus persica var. nectarine Zhongyou No.5) used in this study were sourced from the city of Tai'an, China. All trees were planted for 7 years under standard horticultural practices and were completely productive. In the experiment, there were two treatment groups. The UVB treatments were carried out with $1.44 \mathrm{Kj} \mathrm{m}^{-2} \mathrm{~d}^{-1} \mathrm{UVB}$ radiation, and the young leaves were sprayed with $100 \mu \mathrm{M}$ of $\mathrm{GA}_{3}$ for hormone treatment $[16,34]$. Leaf samples were collected at 3, 6, 9, and $24 \mathrm{~h}$ post-treatment. At each time point, we randomly collected 10 young leaves, which were immediately frozen in liquid nitrogen and stored at $-80^{\circ} \mathrm{C}$ until use. In the tissue expression pattern experiment, we collected samples of five tissues, including root, stem, leaves, shoot tip, and hypocotyl, which were immediately frozen in liquid nitrogen and stored at $-80^{\circ} \mathrm{C}$ until use.

\section{Sequence collection and identification}

The four Rosaceae genome sequences were downloaded from the Genome Database for Rosaceae (GDR) (http://www.rosaceae.org/). To identify putative GRF genes from peach, apple, strawberry, and European pear, initially, the HMM profiles of the GRF domains (PF08879 and PF08880) downloaded from Pfam (http://pfam.xfam.org/) were used to search the database using HMMER3.0 (http://hmmer.janelia.org/). The NCBI CDD (http://www.ncbi.nlm.nih.gov/Structure/cdd/wrpsb.cgi) and SMART (http://smart.embl-heidelberg.de/) were also used to determine the PF08879 and PF08880 domains of GRF, and sequences that lack similar integrity are deleted. Finally, they used the ExPASy proteomics server database to predicted their molecular weights and isoelectric points (http://expasy.org/).

\section{Phylogenetic analysis and sequence alignment}

We used full-length GRF protein sequences (from four Rosaceae species, Arabidopsis, soybean, rice, and poplar) to construct a phylogenetic tree. MEGA 7.0 software was employed to construct phylogenetic 
trees using the neighbor-joining $(\mathrm{NJ})$ method, and the bootstrap test was replicated 1000 times [66]. The conservative motifs were predicted by the MEME online program (http://meme-suite.org/), in which the maximum value of the motif was set to 10 . The intron-exon arrangements were determined by aligning its cDNA sequence with the genomic sequence by TBtools software package. Then, the TBtools software package was used to integrate these analyses [67].

\section{Chromosomal location and collinearity analysis}

MCScanX (http://chibba.pgml.uga.edu/mcscan2/) was used to analyze the WGD/segmental, tandem, proximal, and dispersed duplications to investigate the mechanisms mediating GRF gene family evolution. Based on the statistics of MCScanX, the Ka, Ks and Ka/Ks of duplicated genes were calculated using the KaKs_Calculator 2.0 software [68]. TBtools was used to conduct local analysis of Synteny of four Rosaceae genomes and locate the genes to the chromosomal positions $[67,69]$.

\section{RNA extraction and quantitative RT-PCR analysis}

Total RNA was extracted from different tissues using the RNeasy Plus Mini Kit (Qiagen, Valencia, CA, USA) according to the manufacturer's instructions. To ensure that RNA was DNA-free, reverse transcription of three repeated total RNA samples (approximately $3 \mathrm{mg}$ each) was performed using the PrimeScript RT reagent kit and gDNA Eraser (Perfect Real Time) (Takara Biotechnology, Dalian, China). The primers are listed in Additional File 6 (Supplementary Table S5), and designed using Primer Premier6.22. qRT-PCR was performed using a CFX96 Touch Real-Time PCR Detection System (Bio-Rad, California, USA) with the TB Green ${ }^{\circledR}$ Premix Ex Taq ${ }^{\text {TM }}$ (TaKaRa Biotechnology). The scheme consisted of predenaturation at $95^{\circ} \mathrm{C}$ for $30 \mathrm{~s}$, followed by 40 cycles of denaturation at $95^{\circ} \mathrm{C}$ for $5 \mathrm{~s}$, primer annealing/extension at $60-63^{\circ} \mathrm{C}$ for $30 \mathrm{~s}$, and fluorescence signal detection. Primer specificity was verified by melting curve analysis, which consisted of subjecting the samples to $95^{\circ} \mathrm{C}$ for $10 \mathrm{~s}, 60^{\circ} \mathrm{C}$ for $5 \mathrm{~s}$, and $95^{\circ} \mathrm{C}$ for $0.5 \mathrm{~s}$. Analysis of each sample in triplicate. Then the expression levels of the relative reference genes were determined by $2^{-\triangle \Delta \mathrm{ct}}$ method [70].

\section{Determination of hormone content and growth}

Using liquid-mass spectrometry to determine the content of different hormones at the stem tip tissue was taken on August 7 (vigorous shoot growth) and frozen for storage for the determination of different hormone contents [71]. The measurement of growth index started on June 7 and was measured once per month until the peach tree stopped growing and enters the dormant induction period on October 7. During the measurement, five peach trees with uniform growth in each experimental group were selected, and the length and thickness of the new shoots and the plant height were repeatedly measured, and the average calculated. 


\section{Abbreviations}

GRFs: Growth-regulating factors; UVB: ultraviolet B-rays; $\mathrm{GA}_{3}$ : gibberellin ; IAA: indole-3-acetic acid; TFs: transcription factors; GIFs: growth regulators; HMM: Hidden Markov Model; WGD: whole-genome duplication; Ka: non-synonymous; Ks: synonymous; ABA: abscisic acid; JA: jasmonic acid; TZR: transZeatin-riboside; LREs: light-responsive elements.

\section{Declarations}

\section{Ethics approval and consent to participate}

All the peach materials used in the experiment were collected from Facilities Fruit Tree Laboratory, Shandong Agricultural University. These plant materials are widely used all over the world. and no permits are required for the collection of plant samples. This article did not contain any studies with human participants or animals performed by any of the authors, and did not involve any endangered or protected species.

\section{Consent to publish}

Not applicable.

\section{Availability of data and materials}

The four Rosaceae species genome datasets (Prunus persica, Pyrus communis, Fragaria vesca and Malus domestica) used for identifying the GRF genes in this study were downloaded from the GDR (http://www. rosaceae.org). The other species sequences (Arabidopsis thaliana, Glycine max, Oryza sativa and Populus trichocarpa) were downloaded from the plantTFDB (http://planttfdb.cbi.pku.edu.cn). The RNA-seq data were obtained from our laboratory. The datasets supporting the conclusions of this article were included with in the article and its Supplementary files.

\section{Competing interests}

The authors declare that they have no competing interests.

\section{Funding}

This study was supported by the National Key Research and Developmental Program of China (2018YFD1000104) and $\mathrm{H}_{2} \mathrm{O}_{2}$, NO Mediate PpMAPK13 Regulates Peach Bud Dormancy Release 
Mechanism (ZR2018MC023). These fundings provided the financial support to the research projects, but did not involve in project design, data collection, analysis, or preparation of the manuscript.

\section{Authors' contributions}

XDC, SXL and MYS conceived and designed the experiment. XGM, NW, HJH and DML performed experiments. Li Liu analyzed data. Ling Li made conclusions based on results. MYS wrote the manuscript. All authors read and approved the final manuscript.

\section{Acknowledgements}

We thank all our lab colleagues for their useful discussions and technical assistance. We are very grateful to TopEdit (www.topeditsci.com) for English language editing of this manuscript. We sincerely thank Dr. Chengjie Chen share of TBtools software package.

\section{References}

1. Li M, Li GX, Liu W, Dong XM, Zhang AA. Genome-wide analysis of the NF-Y gene family in peach (Prunus persica L.). BMC Genom. 2019;20:612.

2. Chen M, Tan Q, Sun M, Li D, Fu X, Chen X. et al. Genome-wide Identification of WRKY Family Genes in Peach and Analysis of WRKY Expression During Bud Dormancy. Mol Genet Genomics. 2016;291(3):1319-32.

3. Zhang CH, Ma RJ, Xu JL, Yan J, Guo L, Song J, et al. Genome-wide identification and classification of MYB superfamily genes in peach. Plos One. 2018;13(6):e0199192.

4. Zhang CH, Shangguan LF, Ma RJ, Sun X, Tao R, Guo L. et al. Genome-wide analysis of the AP2/ERF superfamily in peach (Prunus persica). Genet Mol Res. 2012;11(4):4789 - 809.

5. Sun MY, Fu XL, Tan QP, Liu L, Chen M, Zhu CY. et al. Analysis of basic leucine zipper genes and their expression during bud dormancy in peach (Prunus persica). Plant Physiol Biochem. 2016;104:5470.

6. Li H, Dong Q, Zhu X, Zhao Q, Ran K. Genome-wide identification, expression, and interaction analysis for ovate family proteins in peach. Mol Biol Rep. 2019;46(4):3755-64.

7. Van dKE, Kim JH, Kende $\mathrm{H}$. A novel gibberellin-induced gene from rice and its potential regulatory role in stem growth. Plant Physiol. 2000;122(3):695-704.

8. Kim JH, Choi D, Kende H. The AtGRF family of putative transcription factors is involved in leaf and cotyledon growth in Arabidopsis. Plant J. 2003;36(1):94-104.

9. Choi D, Kim JH, Kende H. Whole genome analysis of the OsGRF gene family encoding plant-specific putative transcription activators in rice (Oryza sativa L.). Plant Cell Physiol. 2004;45:897-904. 
10. Treich I, Cairns BR. de los Santos, Brewster E, Carlson M, SNF11, a new component of the yeast SNF-SWI complex that interacts with a conserved region of SNF2, Mol. Cell Biol. 1995;15: 4240-8.

11. Chen F, Yang YZ, Luo XF, Zhou WG, Dai YJ, Zheng C. et al. Genome-wide identification of GRF transcription factors in soybean and expression analysis of GmGRF family under shade stress. BMC Plant Biol. 2019;19(1):269.

12. Zhang DF, Bo L, Jia GQ, Zhang TF, Dai JR, Li JS. et al. Isolation and characterization of genes encoding GRF transcription factors and GIF transcriptional coactivators in maize (Zea mays L.). Plant Sci. 2008;175(6):809-17.

13. Lee BH, Jeon JO, Lee MM, Kim JH. Genetic interaction between GROWTH-REGULATING FACTOR and CUP-SHAPED COTYLEDON in organ separation. Plant Signal Behav. 2015;10(2):e988071.

14. Debernardi JM, Mecchia MA, Vercruyssen L, Smaczniak C, Kaufmann K, Inze D. et al. Posttranscriptional control of GRF transcription factors by microRNA miR396 and GIF co-activator affects leaf size and longevity. Plant J. 2014;79(3):413-26.

15. Horiguchi G, Kim G-T, Tsukaya H. The transcription factor AtGRF5 and the transcription coactivator AN3 regulate cell proliferation in leaf primordia of Arabidopsis thaliana. Plant J. 2005;43(1):68-78.

16. Ma JQ, Jian HJ, Yang B, Lu K, Zhang AX, Liu P. et al. Genome-wide analysis and expression profiling of the GRF gene family in oilseed rape (Brassica napus L.). Gene. 2017;620:36-45.

17. Kim JH, Lee BH. GROWTH-REGULATING FACTOR4 of Arabidopsis thaliana is required for development of leaves, cotyledons, and shoot apical meristem. J Plant Biol. 2006;49(6):463-8.

18. Omidbakhshfard MA, Proost S, Fujikura U, Mueller-Roeber B. Growth-regulating factors (GRFs): a small transcription factor family with important functions in plant biology. Mol Plant. 2015;8(7):998-1010.

19. Lee BH, Ko JH, Lee S, Yi L, Pak JH, Kim JH. The Arabidopsis GRF-INTERACTING FACTOR gene family performs an overlapping function in determining organ size as well as multiple developmental properties. Plant Physiol. 2009;151(2):655-68.

20. Schommer C, Debernardi JM, Bresso EG, Rodriguez RE, Palatnik JF. Repression of Cell Proliferation by miR319-regulated TCP4. Mol Plant. 2014;7(10):1533-44.

21. Hoe Kim J, Tsukaya H. Regulation of plant growth and development by the GROWTH-REGULATING FACTOR and GRF-INTERACTING FACTOR duo. J Exp Bot. 2015;66(20):6093-107.

22. Liang G, He H, Li Y, Wang F, Yu D. Molecular mechanism of microRNA396 mediating pistil development in Arabidopsis. Plant Physiol. 2014;164(1):249-58.

23. Lee BH, Kim JH. Spatio-temporal distribution patterns of GRF-INTERACTING FACTOR expression and leaf size control. Plant Signal Behav. 2014;9(9):e29697.

24. Yang F, Liang G, Liu D, Yu D. Arabidopsis MiR396 mediates the development of leaves and flowers in transgenic tobacco. J Plant Biol. 2009;52(5):475-81.

25. Liu D, Song Y, Chen Z, Yu D. Ectopic expression of miR396 suppresses GRF target gene expression and alters leaf growth in Arabidopsis. Physiol Plant. 2009;136(2):223-36. 
26. Rodriguez RE, Mecchia MA, Debernardi JM, Schommer C, Weigel D, Palatnik JF. Control of cell proliferation in Arabidopsis thaliana by microRNA miR396. Development. 2010;137(1):103-12.

27. Hewezi T, Maier TR, Nettleton D, Baum TJ. The Arabidopsis microRNA396-GRF1/GRF3 regulatory module acts as a developmental regulator in the reprogramming of root cells during cyst nematode infection. Plant Physiol. 2012;159:321-35.

28. Vercruyssen L, Tognetti VB, Gonzalez N. Van Dingenen J, De Milde L, Bielach A,et al. GROWTH REGULATING FACTOR5 stimulates Arabidopsis chloroplast division, photosynthesis, and leaf longevity. Plant Physiol. 2015;167: 817-32.

29. Fina J, Casadevall R, AbdElgawad H, Prinsen E, Markakis MN, Beemster GTS, Casati P. UV-B Inhibits Leaf Growth Through Changes in Growth Regulating Factors and Gibberellin Levels. Plant Physiol. 2017;174(2):1110-26.

30. Casadevall R, Rodriguez RE, Debernardi JM, Palatnik JF, Casati P. Repression of growth regulating factors by the microRNA396 inhibits cell proliferation by UV-B radiation in Arabidopsis leaves. Plant Cell. 2013;25:3570-83.

31. Chen F, Yang Y, Luo X, Zhou W, Dai Y, Zheng C. et al. Genome-wide identification of GRF transcription factors in soybean and expression analysis of GmGRF family under shade stress. BMC Plant Biol. 2019;19(1):269.

32. Zheng Y, Crawford GW, Chen X. Archaeological evidence for peach (Prunus persica) cultivation and domestication in china. Plos One. 2014;9(9):e106595.

33. Wang XX, Fu XL, Chen M, Huan L, Liu WH, Qi YH. et al. Ultraviolet B irradiation influences the fruit quality and sucrose metabolism of peach (Prunus persica L.). Environ Exp Bot. 2018;153:286-301.

34. Li S, Shao Z, Fu X, Xiao W, Li L, Chen M. et al. Identification and characterization of Prunus persica miRNAs in response to UVB radiation in greenhouse through high-throughput sequencing. BMC Genom. 2017;18(1):938.

35. Yu N, Li D, Tan Q, Zhang H, Gao D. Effect of UVB radiation on assimilate translocation and distribution in fruiting shoot of protected peach. Appl Environment Biol. 2013;19(1):157 - 63.

36. Wang F, Qiu N, Qian D, Li J, Zhang Y, Li H. et al. Genome-wide identification and analysis of the growth-regulating factor family in Chinese cabbage (Brassica rapa L. ssp. pekinensis). BMC Genom. 2014;15:807.

37. Zhang J, Li Z, Jin J, Xie X, Zhang H, Chen Q. et al. Genome-wide identification and analysis of the growth-regulating factor family in tobacco (Nicotiana tabacum). Gene. 2018;639:117 - 27.

38. Shang S, Wu C, Huang C, Tie W, Yan Y, Ding Z. et al. Genome-wide analysis of the GRF family reveals their involvement in abiotic stress response in cassava. Genes. 2018;9(2):110.

39. Cao Y, Han Y, Jin Q, Lin Y, Cai Y. Comparative Genomic Analysis of the GRF Genes in Chinese Pear (Pyrus bretschneideri Rehd), Poplar (Populous), Grape (Vitis vinifera), Arabidopsis and Rice (Oryza sativa). Front Plant Sci. 2016;7:1750.

40. Ma JQ, Jian HJ, Yang B, Lu K, Zhang AX, Liu P. et al. Genome-wide analysis and expression profiling of the GRF gene family in oilseed rape (Brassica napus L.). Gene. 2017;620:36-45. 
41. Gonzalez N, Vanhaeren H, Inzé D. Leaf size control: complex coordination of cell division and expansion. Trends Plant Sci. 2012;17(6):332 - 40.

42. Lescot M, Dehais P, Thijs G, Marchal K, Moreau Y, Peer VY. et al. PlantCARE, a database of plant cisacting regulatory elements and a portal to tools for in silico analysis of promoter sequences. Nucleic Acids Res. 2002;30:325-7.

43. Gangappa SN, Maurya JP, Yadav V, Chattopadhyay S. The regulation of the Z- and G-box containing promoters by light signaling components, SPA1 and MYC2, in Arabidopsis. PLoS One. 2013;8(4):e62194.

44. Roy S, Choudhury SR, Singh SK, Das KP. Functional analysis of light-regulated promoter region of AtPold gene. Planta. 2012;235(2):411 - 32.

45. Chattopadhyay S, Ang LH, Puente P, Deng XW, Wei N. Arabidopsis bZIP protein HY5 directly interacts with light-responsive promoters in mediating light control of gene expression. Plant Cell. 1998 May;10(5):673 - 83.

46. Li W, Bai Z, Pei T, Yang D, Mao R, Zhang B. et al. SmGRAS1 and SmGRAS2 Regulate the Biosynthesis of Tanshinones and Phenolic Acids in Salvia miltiorrhiza. Front Plant Sci. 2019;10:1367.

47. Zhang LF, Li WF, Han SY, Yang WH, Qi LW. cDNA cloning, genomic organization and expression analysis during somatic embryogenesis of the translationally controlled tumor protein (TCTP) gene from Japanese larch (Larix leptolepis). Gene. 2013;529(1):150-8.

48. Xin S, Tao C, Li H. Cloning and Functional Analysis of the Promoter of an Ascorbate Oxidase Gene from Gossypium hirsutum. PLoS One. 2016;11(9):e0161695.

49. Sakai T, Takahashi Y, Nagata T. Analysis of the promoter of the auxin-inducible gene, parC, of tobacco. Plant Cell Physiol. 1996;37(7):906 - 13.

50. Guerriero G, Martin N, Golovko A, Sundström JF, Rask L, Ezcurra I. The RY/Sph element mediates transcriptional repression of maturation genes from late maturation to early seedling growth. New Phytol. 2009;184(3):552 - 65.

51. Li H, Han X, Qiu W, Xu D, Wang Y, Yu M, Hu X, Zhuo R. Identification and expression analysis of the GDSL esterase/lipase family genes, and the characterization of SaGLIP8 in Sedum alfredii Hance under cadmium stress. PeerJ. 2019;7:e6741.

52. Li S, Gao F, Xie K, Zeng X, Cao Y, Zeng J. et al. The OsmiR396c-OsGRF4- OsGIF1 regulatory module determines grain size and yield in Rice. Plant Biotechnol J. 2016;14(11):2134-46.

53. Velasco R, Zharkikl A, Affourtit J, Dhingra A, Cestaro A. et al. The genome of the domesticated apple (Malus6domestica Borkh.). Nat Genet. 2010;42:833-9.

54. Cao ZH, Zhang SZ, Wang RK, Zhang RF, Hao YJ. Genome wide analysis of the apple MYB transcription factor family allows the identification of MdoMYB121 gene confering abiotic stress tolerance in plants. PLoS One. 2013;8(7):e69955.

55. Chen XD. The effects of ultraviolet-B radiation intensity and different plasic film on development characteristics of peach flower and fruit in protected culture. Shandong Agriculture University, Horticulture and Engineering college: Master Thesis; 2009. 
56. Casati $\mathrm{P}, \mathrm{Walbot} \mathrm{V}$. Gene expression profiling in response to ultraviolet radiation in maize genotypes with varying flavonoid content. Plant Physiol. 2003;132:1739-54.

57. Wu ZJ, Wang WL, Zhuang J. Developmental processes and responses to hormonal stimuli in tea plant (Camellia sinensis) leaves are controlled by GRF and GIF gene families. Funct Integr Genomics. 2017;17(5):503 - 12.

58. Liu J, Hua W, Yang HL, Zhan GM, Li RJ, Deng LB. et al. The BnGRF2 gene (GRF2-like gene from Brassica napus) enhances seed oil production through regulating cell number and plant photosynthesis. J Exp Bot. 2012;63(10):3727-40.

59. Jérémie B, Ghazanfar Abbas K, Jean-Philippe C, Pilar BS, Juan Manuel D, Ramiro R. et al. miR396 affects mycorrhization and root meristem activity in the legume Medicago truncatula. Plant $\mathrm{J}$. 2013;74(6):920 - 34.

60. Kuijt SJ, Greco R, Agalou A, Shao JT, Hoen CC, Overnas E. et al. Interaction between the GROWTHREGULATING FACTOR and KNOTTED1-LIKE HOMEOBOX families of transcription factors. Plant Physiol. 2014;164(4):1952-66.

61. He Y, Zhang Y, Chen L, Wu C, Luo Q, Zhang F. et al. Member of the 14-3-3 Gene Family in Brachypodium distachyon, BdGF14d, Confers Salt Tolerance in Transgenic Tobacco Plants. Front Plant Sci. 2017;8:340.

62. Chen Y, Zhou X, Chang S, Chu Z, Wang H, Han S. et al. Calcium-dependent protein kinase 21 phosphorylates 14-3-3 proteins in response to ABA signaling and salt stress in rice. Biochem Biophys Res Commun. 2017;493(4):1450-6.

63. Kaundal A, Ramu VS, Oh S, Lee S, Pant B, Lee HK. et al. GENERAL CONTROL NONREPRESSIBLE4 Degrades 14-3-3 and the RIN4 Complex to Regulate Stomatal Aperture with Implications on Nonhost Disease Resistance and Drought Tolerance. Plant Cell. 2017;29(9):2233-48.

64. Yang L, You J, Wang Y, Li J, Quan W, Yin M. et al. Systematic analysis of the G-box Factor 14-3-3 gene family and functional characterization of GF14a in Brachypodium distachyon. Plant Physiol Biochem. 2017;117:1-11.

65. Liu Z, Jia Y, Ding Y, Shi Y, Li Z, Guo Y. et al. Plasma Membrane CRPK1-Mediated Phosphorylation of 14-3-3 Proteins Induces Their Nuclear Import to Fine-Tune CBF Signaling during Cold Response. Mol Cell. 2017;66(1):117-28.

66. Tamura K, Stecher G, Peterson D, Filipski A, Kumar S. Mega6: molecular evolutionary genetics analysis version 6.0. Mol Biol Evol. 2013;30(4):2725-9.

67. Chen C, Xia R, Chen H, He Y. TBtools, a toolkit for biologists integrating various HTS-data handling tools with a user-friendly interface. bioRxiv. 2018. https://doi.org/10.1101/289660.

68. Zhang Z, Li J, Zhao X-Q, Wang J, Wong GK-S, Yu J. KaKs_Calculator: calculating Ka and Ks through model selection and model averaging. Genom Proteom Bioinf. 2006;4(4):259 - 63.

69. Xie T, Chen C, Li C, Liu J, Liu C, He Y. Genome-wide investigation of WRKY gene family in pineapple: evolution and expression profiles during development and stress. BMC Genom. 2018;19:490. 
70. Livak KJ, Schmittgen TD. Analysis of relative gene expression data using real-time quantitative PCR and the $2^{-\Delta \Delta C T}$ Method. Methods. 2001;25:402-8.

71. Mikiko K, Tomoe KN, Hirokazu K, Kentaro T, Takeshi K, Masaharu M. et al. Highly Sensitive and HighThroughput Analysis of Plant Hormones Using MS-Probe Modification and Liquid ChromatographyTandem Mass Spectrometry: An Application for Hormone Profiling in Oryza sativa. Plant Cell Physiol. 2009;50(7):1201-14.

\section{Tables}

Due to technical limitations, the tables are provided in the Supplementary Files section.

\section{Figures}




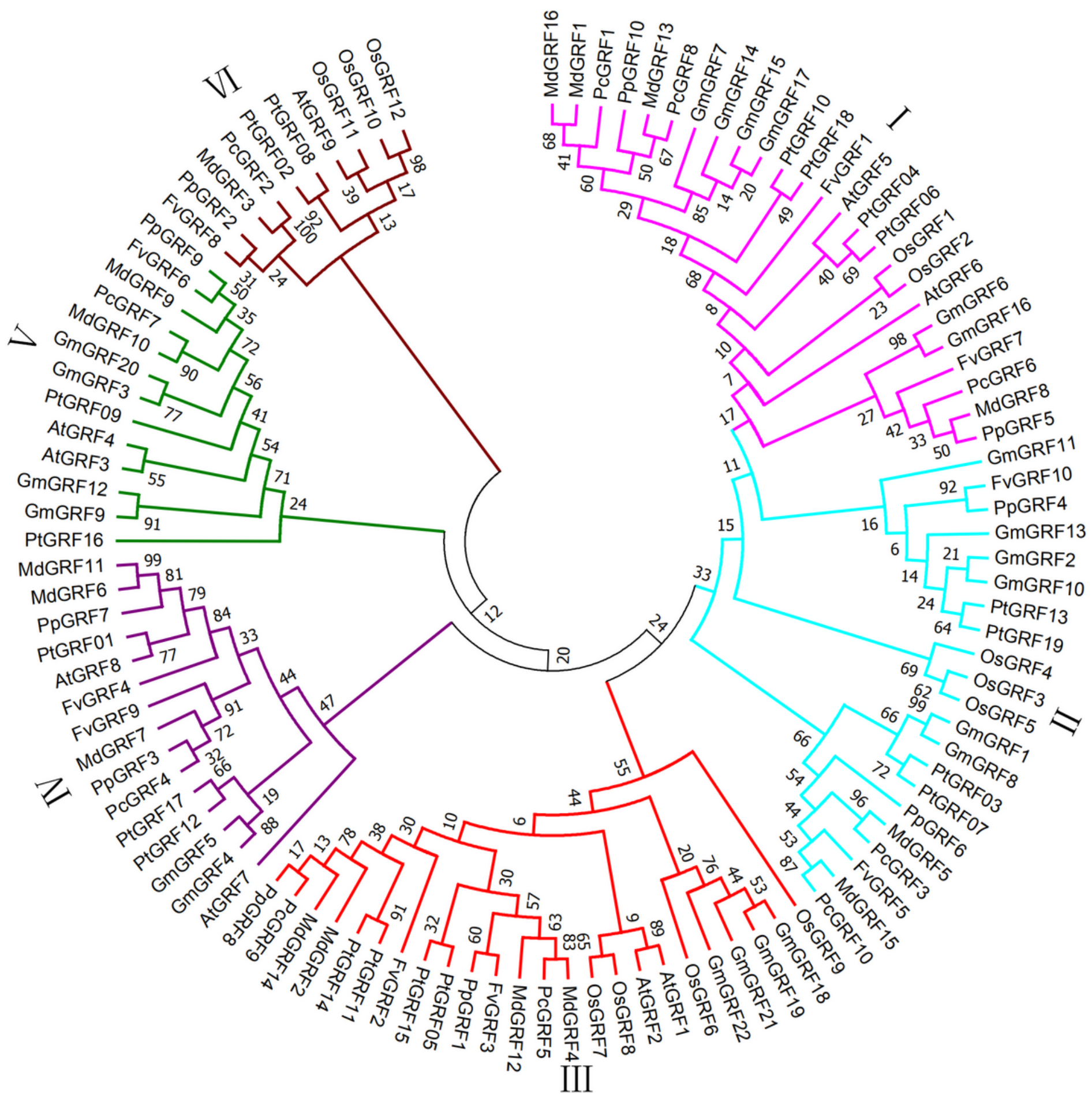

Figure 1

Phylogenetic analysis of GRF from Arabidopsis, Oryza, Populus, Glycine max, Peach, European pear, Strawberry and Apple. Using the Neighbor-Joining method to construct the phylogenetic tree. Six subfamilies are shown in different colored 

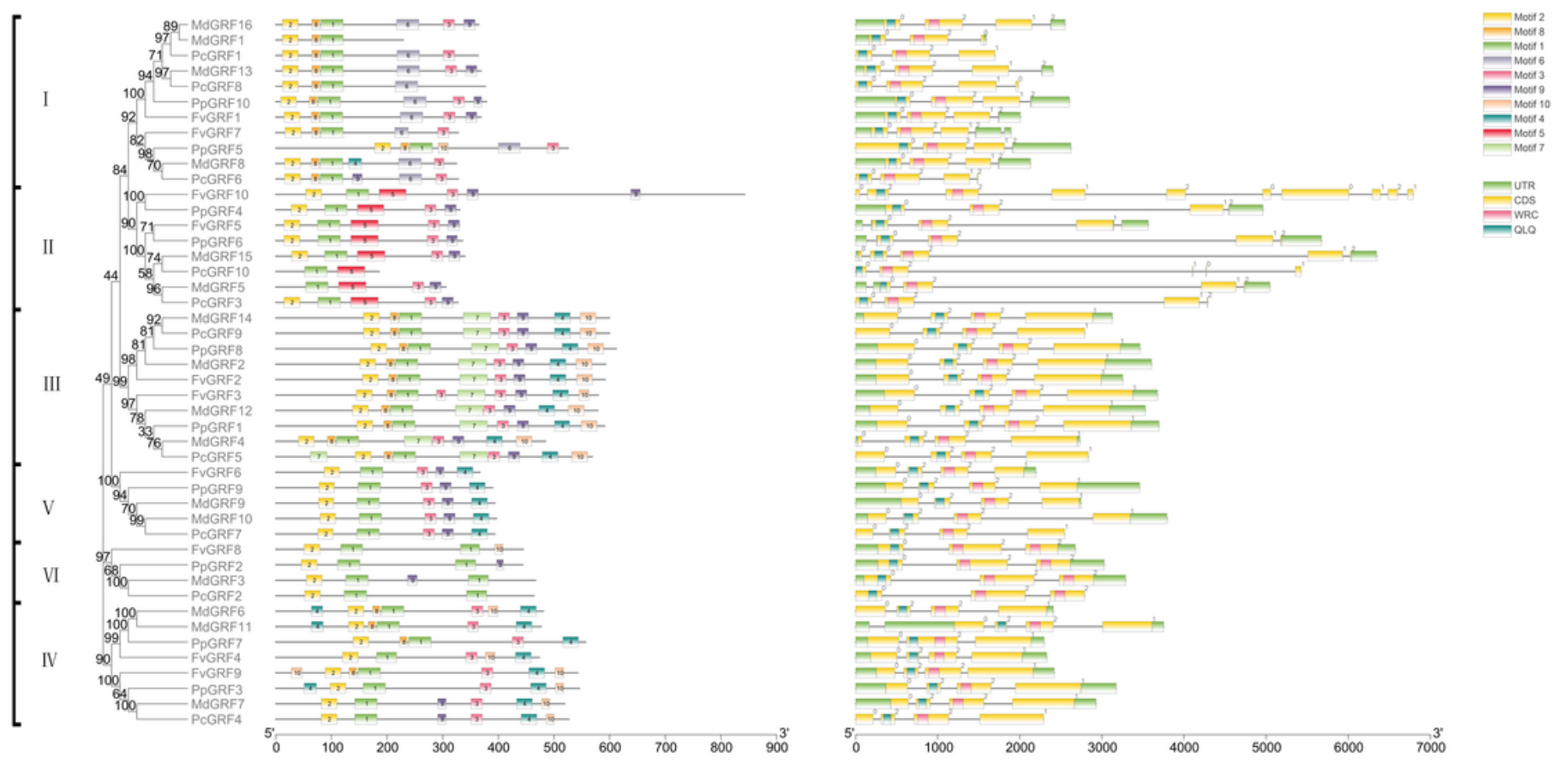

Figure 2

Phylogenetic relationships, gene structures and motif structure in GRF genes from four Rosaceae species (Peach, European pear, Strawberry and Apple). (a)The phylogenetic tree was constructed based on the full-length sequences of GRF proteins using MEGA7.0. (b) Schematic representation of the 10 conserved motifs in GRF proteins. Members of same group are arranged sequentially according to phylogenetic classification. The conserved motifs labeled with different colors boxes. The length of the protein can be estimated using the scale at the bottom. (c) Exon-intron structures of the GRF genes. Green boxes indicate untranslated 5'- and 3'-regions; yellow boxes indicate exons; and black lines indicate introns. The QLQ and WRC domains are highlighted by blue and red boxes. The number indicates the phases of the corresponding introns. The length of the nucleic acid can be estimated using the scale at the bottom 


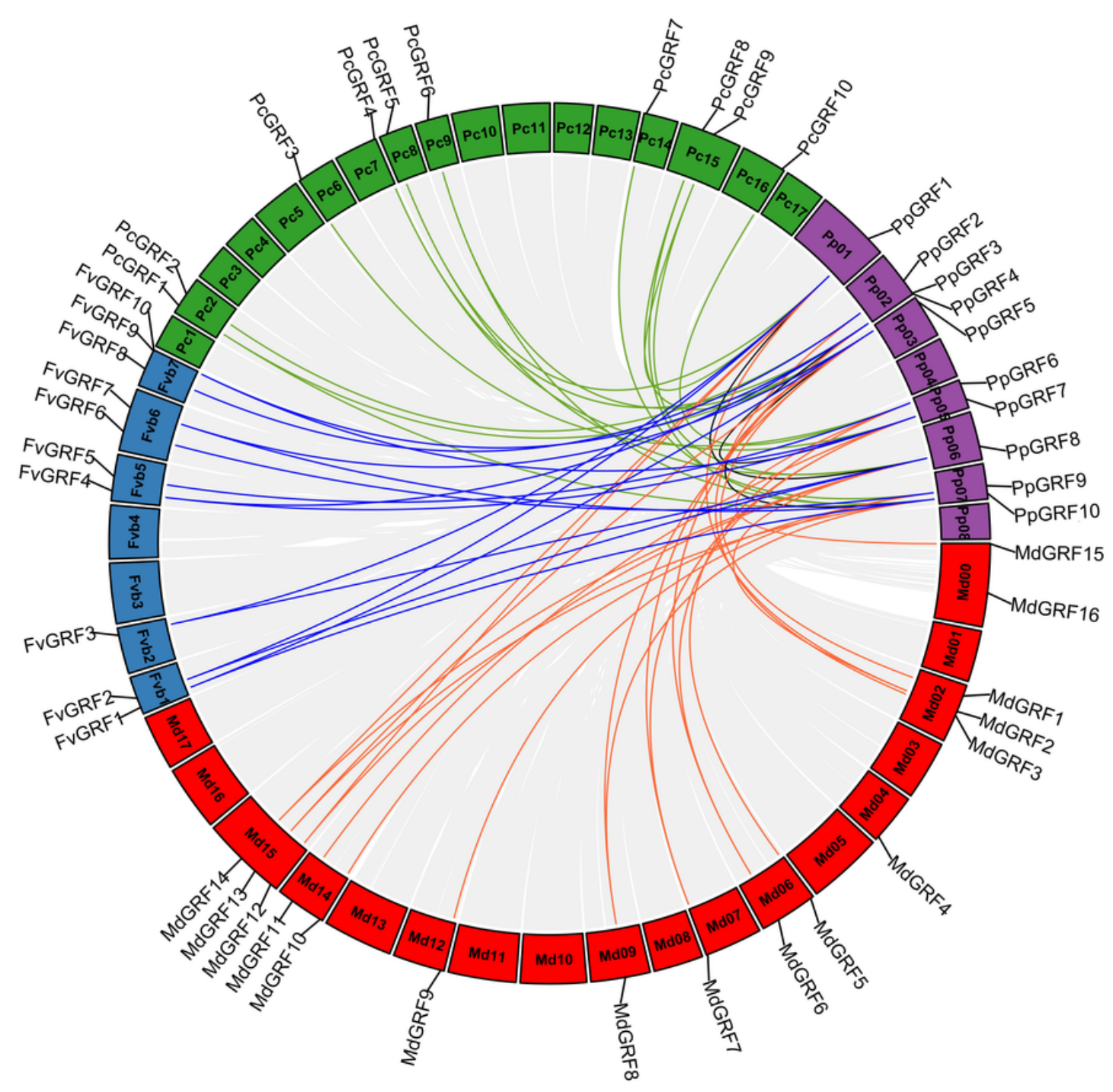

Figure 3

Gene location and collinearity analysis of the GRF genes between peach and three Rosaceae plants (European pear, Strawberry and Apple). Gray bars in the background indicate the collinear blocks within the peach and other plant genomes. Different genomes are indicated by group-specific color. Syntenic gene pairs are highlighted by lines in different colors 

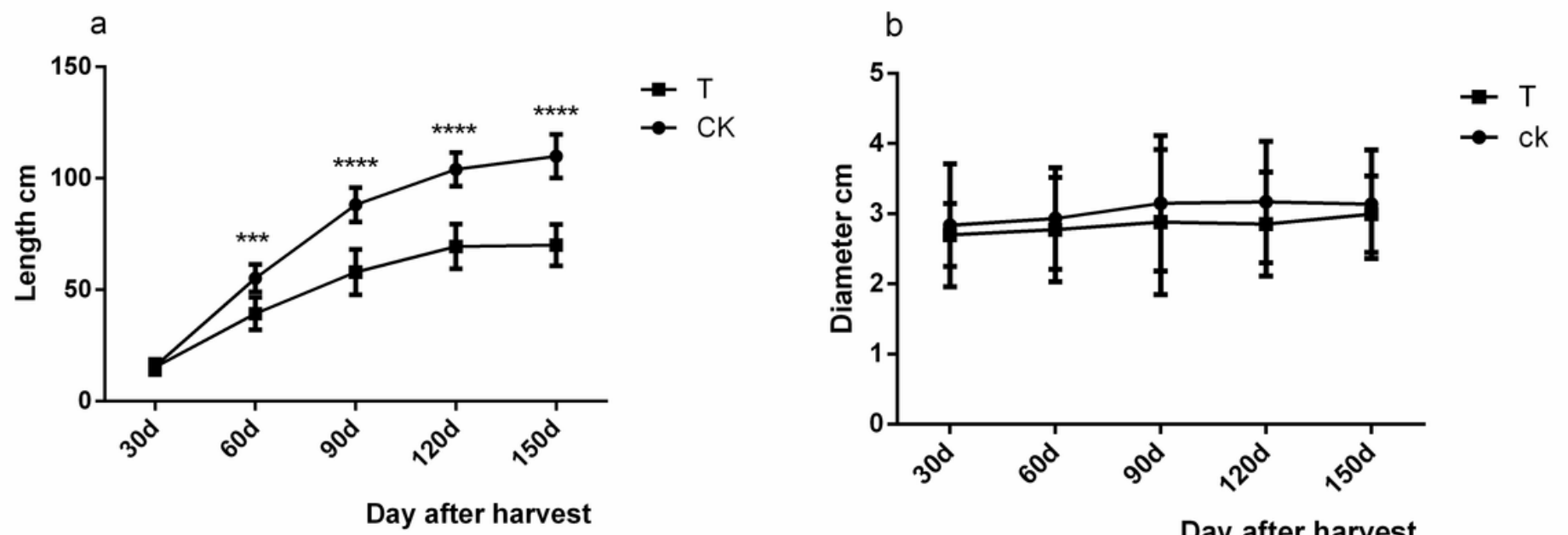

\section{Figure 4}

Effects of UVB treatment on the growth of peach new shoots. a. new shoot length, b. new shoot thickness. The data indicate variation after harvest from 7 June 2019 to 7 October 2019. Values are means $(n=3)$ and error bars represent the standard deviation. 

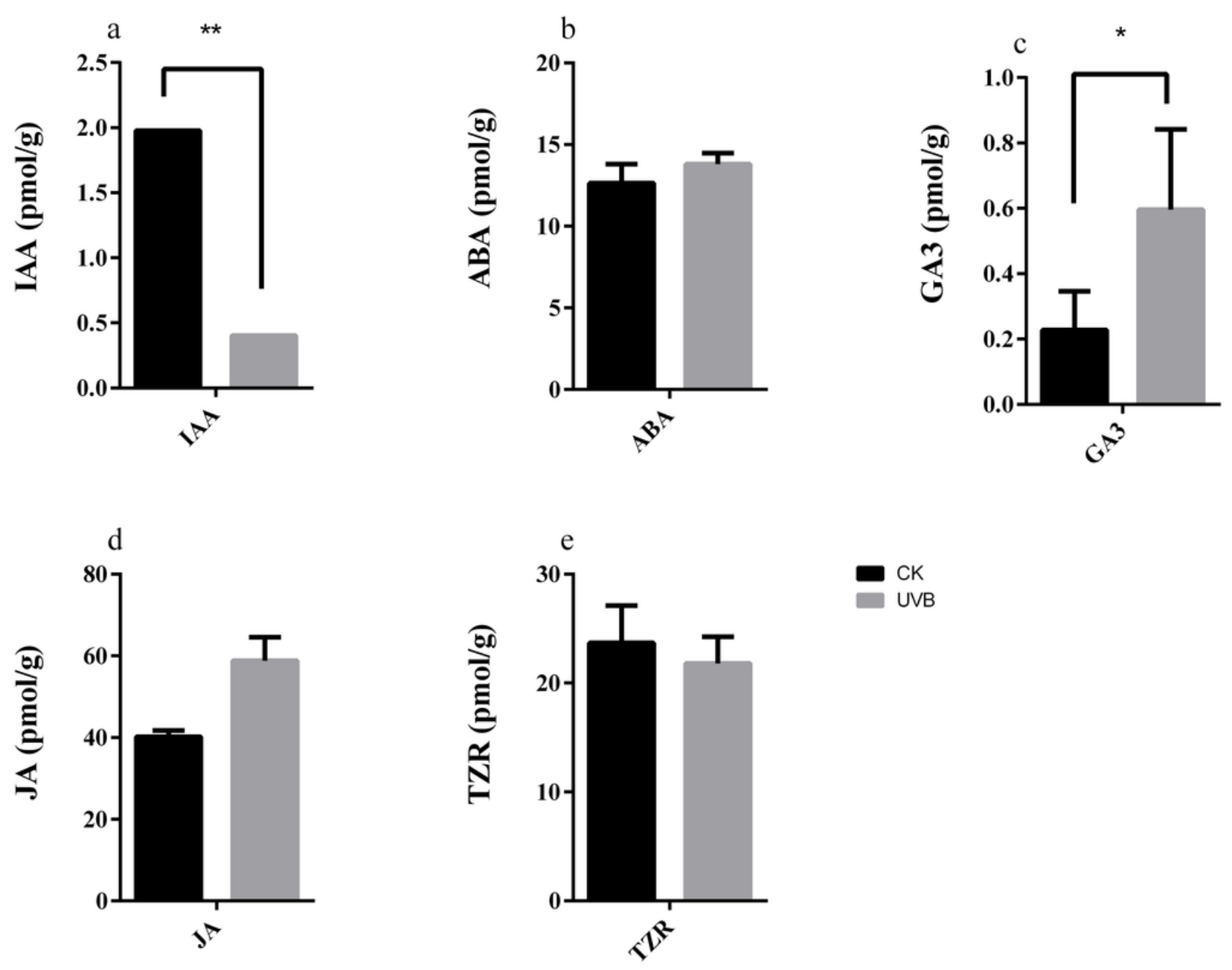

Figure 5

Contents of different hormones in stem apex. a: IAA; b: ABA; c: GA3; d: JA; e: TZR. Data is presented as the means $\pm S D, n=3$. Different Asterisks above bars denote statistical significance according to one-way ANOVA and Duncan's test. 


\section{QLQ}

WRC

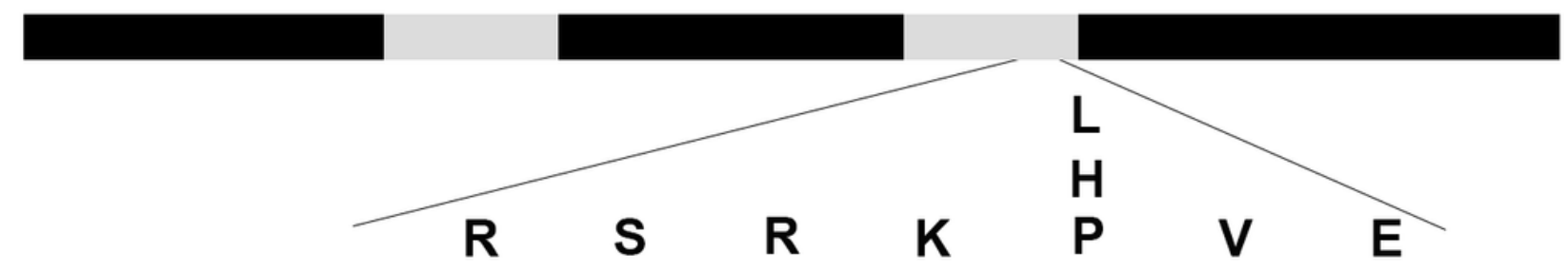

Ppgrf1 5'- C C G

Ppgrf2 5'- U C G G U U C A A

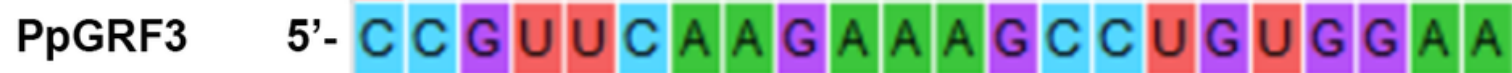

Ppgrf4 5'- U C G U U U C A A

Ppgrf5 5'- C C G U U C A A

Ppgrf6 5'- C C G U U C A A

Ppgrf7 5'- C C G U U U C A A

Ppgrf8 5'- G C G G U C A A $G$ A A A $G$ G C C U G U G G A A

Ppgrf9 5'- C C G U U C A A

Ppgrf10 5'- C C G U U C A A

miR396a $U$ GCAAAGU

miR396b UUCAAGUUCUUUCG - ACA C C UU -5'

Figure 6

Prediction of the miR396 target site. Scheme representing the GRF genes. The interaction of the ten GRFs from peach with miR396 is shown. QLQ and WRC indicate the conserved domains that define the GRF family 

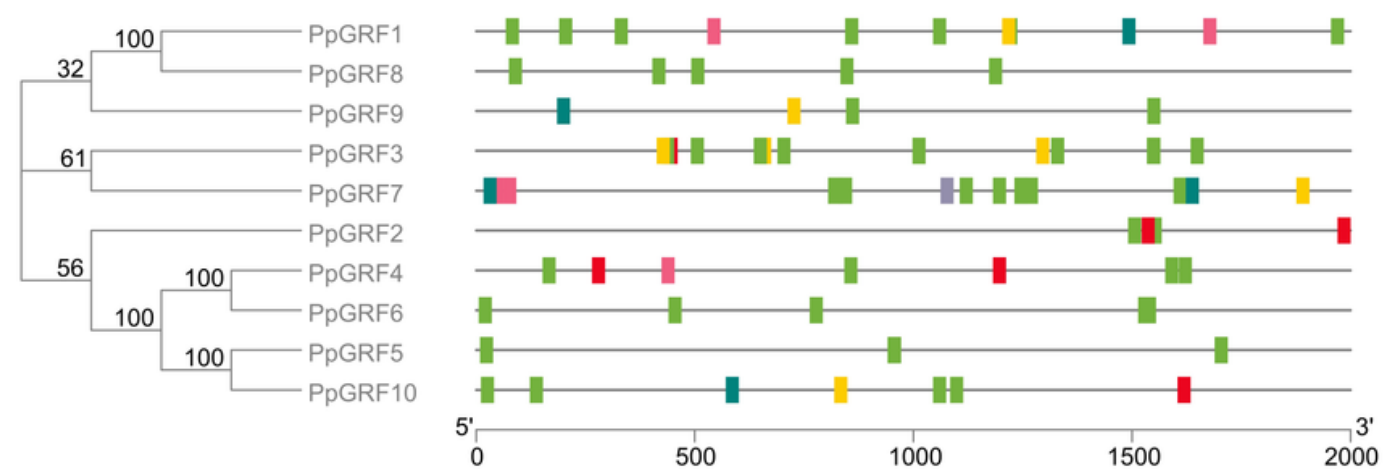

Figure 7

Schematic model of six cis-elements in the promoter sequences of PpGRF genes. Members of GRF are arranged sequentially according to phylogenetic classification. The length of the nucleic acid can be estimated using the scale at the bottom

PpGRF1

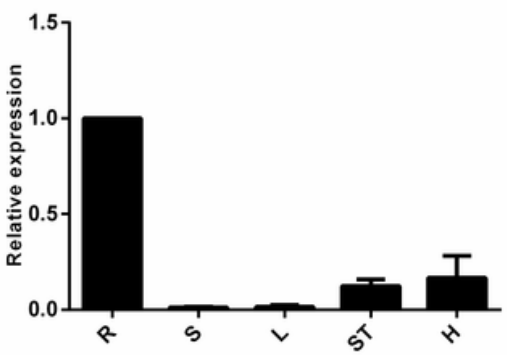

PpGRF4

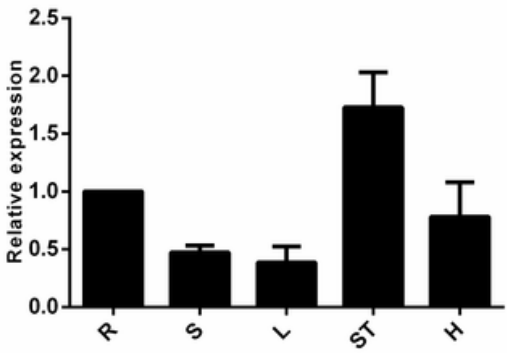

PpGRF7

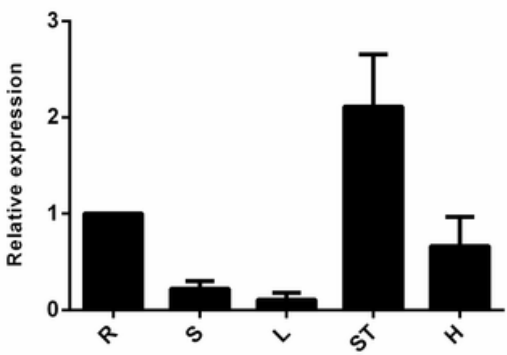

PpGRF2

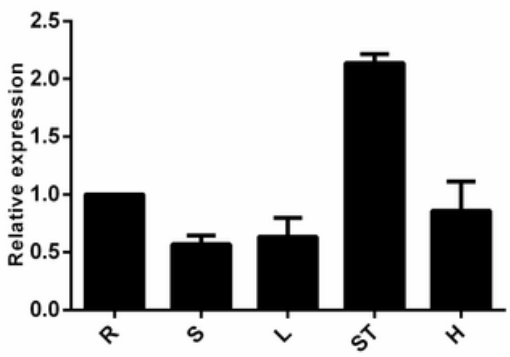

PpGRF5

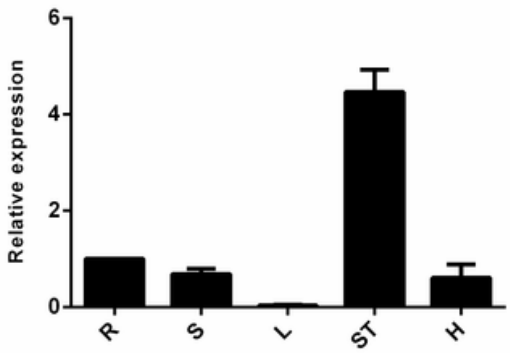

PpGRF9

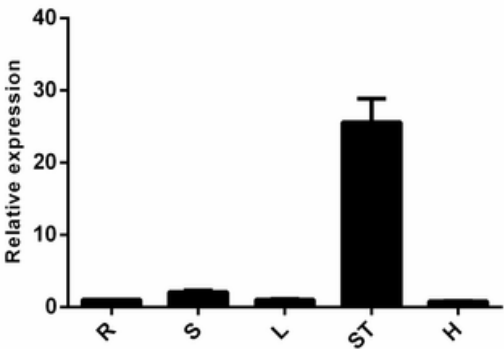

PpGRF3

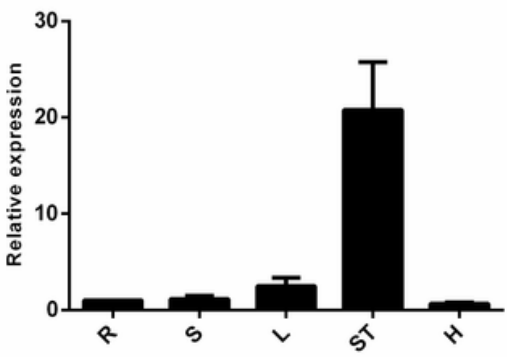

PpgRF6

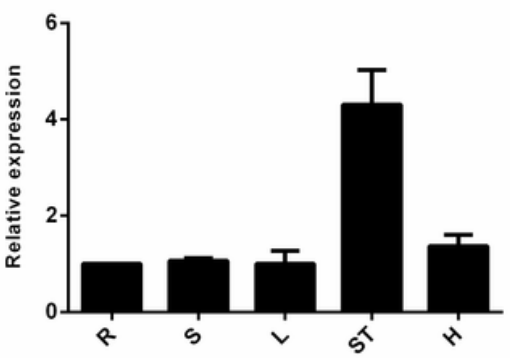

PpGRF10

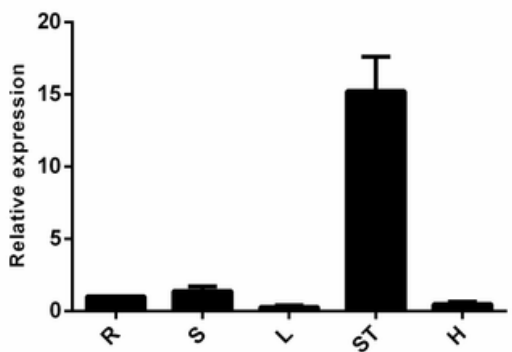

Figure 8 
Expression profiles of PpGRF genes in sixs peach tissues. Root (R), stem (S), leaves (L), shoot tip (ST) and hypocotyl $(\mathrm{H})$. The $\mathrm{x}$-axis represents different peach organs. The $y$-axis represents the relative expression levels of PpGRF genes compared with actin. The expressions of PpGRF genes in root were normalized to 1. Error bars represent standard deviations of the means of three independent replicates.
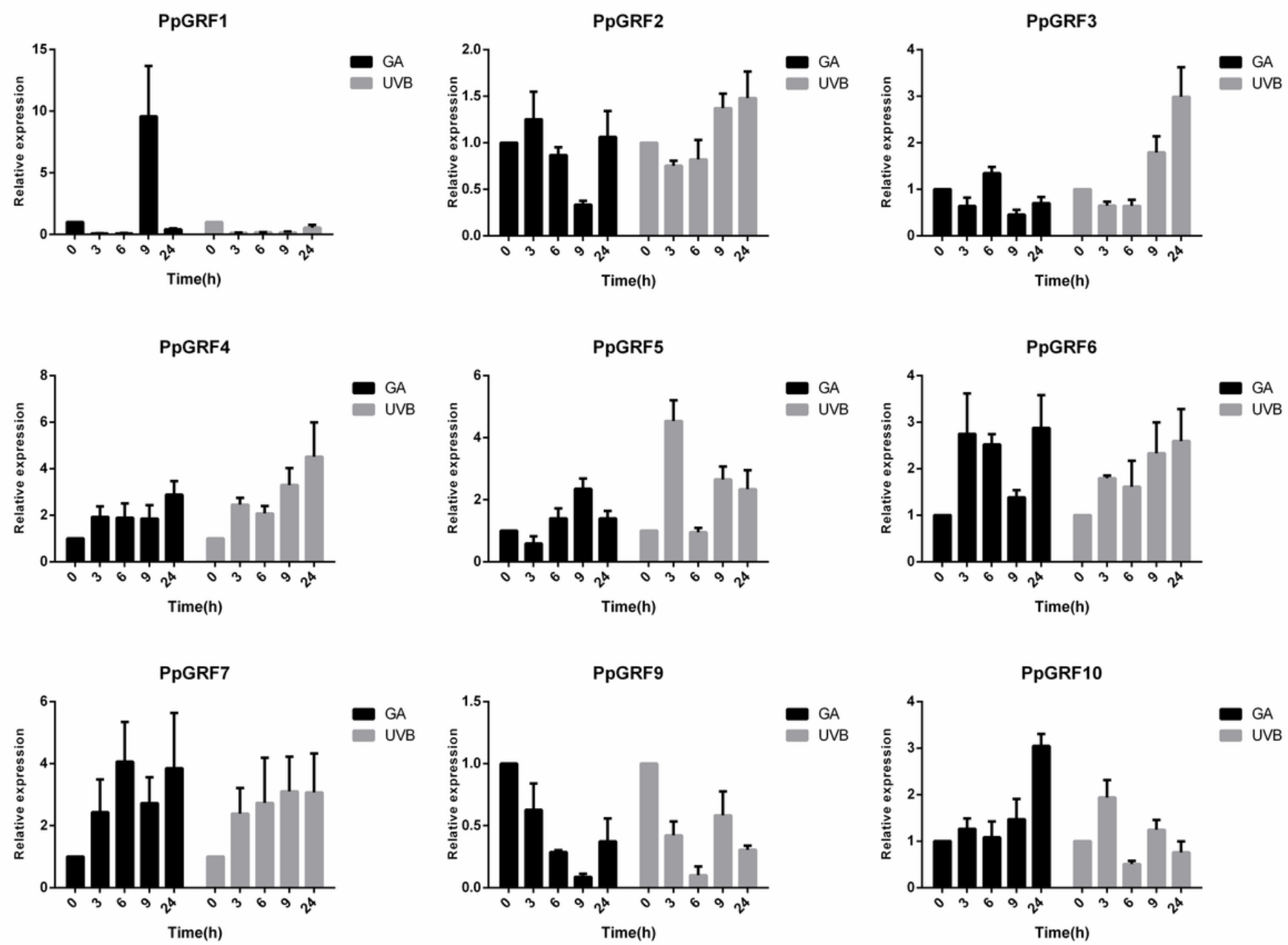

Figure 9

Relative expression analysis of the PpGRFs under UVB and GA3 treatment. The $x$-axis represents different time. The $y$-axis represents the relative expression levels of PpGRF genes compared with actin. Data presented are the means $\pm S D$ of three replicates.

\section{Supplementary Files}

This is a list of supplementary files associated with this preprint. Click to download.

- Tab1.xls

- Additionalfile1Tables1.xls 
- Additionalfile2Tables2.xls

- Additionalfile3TableS3.xlsx

- AdditionalFile4FigureS1.jpg

- Additionalfile5TableS4.doc

- Additionalfile6Tables5.xls 